the ratio is $>1 / \sqrt{2}$. The penetration distance, the depth to which a small magnetic field penetrates into a superconducting metal, is given by $m c^{2} / 4 \pi n e^{2}$ and is large for a large electron mass $m$ and a small electron concentration $n$ ( $c$ is the velocity of light and $e$ the electron charge). The coherence length $\xi \approx 0.2$ $\left(\hbar v_{F} / k_{B} T_{c}\right)$ is roughly the size of the Cooper electron pairs which arise in a metal after the superconducting transition and form the superconducting electron liquid ( $h$ and $k_{\mathrm{B}}$ are the Planck and Boltzmann constants, respectively). It is small for a large $T_{c}$ and a small Fermi velocity $v_{F}$. One thus expects $\lambda$ to be large and $\xi$ small in poorly conducting "metals" with rather high transition temperatures such as organic or oxide superconductors, so these materials should be Type II superconductors characterized by $\lambda / \xi>1 / 2$.

There have been only few reported attempts to determine $\lambda$ in the quasi two-dimensional organic superconductors. They give values on the order of $10^{3} \AA$ in the $a b$-plane. The coherence length $\xi_{a b}$ in the same plane has been found to be on the order of $10^{2} \AA$, and $\xi_{\mathrm{ac}} \approx \xi_{\mathrm{bc}} \approx 10 \AA$. These values are fully consistent with the normal state parameters described above.

\section{Non-magnetic impurities}

At present, the nature of the interactions leading to the formation of superconducting pairs in organic superconductors remains unknown although ordinary BCS superconductivity with the usual phonon mediated pairing can still be considered as a likely interpretation. However, more exotic pairing mechanisms have been proposed, in parti- cular pairing mediated by spin excitations instead of phonons.

The only feature which definitely points to an exceptional property is the extremely strong dependence of transition temperatures on the concentration of non-magnetic impurities. The most prominent demonstration of this sensitivity is shown by the $\beta$-(ET) $I_{3}$ salt. Its two phases, $\beta_{L}$ and $\beta_{H}$, have transition temperatures of 1.5 and $8 \mathrm{~K}$ respectively, differing by more than a factor of five. Measurements of several normal state properties, including paramagnetic susceptibility and the proton magnetic resonance relaxation rate, nevertheless show that the electron systems in these two phases have very similar characteristics. This is also confirmed by the observation of approximately the same values for the gradients of the $\rho$ versus $T^{2}$ plots for the two phases, confirming that the strength of the electron-electron interaction remains roughly constant. The only difference is the presence in the $\beta_{L}$-phase of an additional electron scattering mechanism, absent in the $\beta_{H^{-}}$-phase, that is associated with partial disordering of the crystal lattice. The most notable manifestation of this disordering is a residual resistance $\rho_{0}$ some 20-30 times larger in $\beta_{L}$ than in $\beta_{H}$. It is well known that scattering on such non-magnetic defects formed by lattice disordering does not affect conventional superconductivity. However, the high sensitivity of the superconducting transition temperature to the presence of non-magnetic impurities (which is a characteristic feature of all known organic superconductors) as well as the observation that the nuclear spin-lattice relaxation rate of (TMTSF) 2
$\mathrm{ClO}_{4}$ follows a power law temperature dependence in the superconducting phase point to unusual mechanisms for superconductivity.

\section{Conclusions}

This brief review of organic superconductors demonstrates that truely high temperature superconductivity was found down a different path. Does this mean that all our efforts have turned out to be useless? I think not. The presence in solid state physics of the novel objects, namely highly anisotropic organic metals and superconductors, has resulted in the observation of a number of new phenomena, such as the Peierls instability, charge and spin density waves, phonon-assisted electron localization and so on. All this has contributed to the clarification of some old concepts concerning both the normal and superconducting state in metals, and to the formulation of some new ones. Some of these concepts have turned out to be useful for understanding, in particular, various properties of the high- $T_{c}$ oxide superconductors, which also have layered crystal structures and many other features in common with their organic counterparts.

Finally, and perhaps most important of all, I believe that we have not heard the last word on organic superconductivity: we should anticipate the appearance of new materials, new properties and new ideas.

\section{REFERENCES}

[1] Ribault M. et al., J. Physique Lett. 41 (1980) L607.

[2] Jérome D. and Bechgaard K., Europhys. News 14 (1983) 5, 7.

[3] Jérome D., Phys. Bull. 37 (1986) 171.

\title{
Science and Technology of Conducting Polymers
}

\author{
EIW-6, Europhysics Industrial Workshop
}

Lofthus, Norway, 28-31 May 1990

The sixth in the series of Europhysics Industrial Workshops on the science and technology of conducting polymers was held in Lofthus, Norway on 28-31 May 1990. It was organized by W.R. Salaneck, Linköping University, Sweden, D.T. Clark, I.C.I., England, and E.J. Samuelsen, Trondheim University, Norway and was attended by almost 60 participants, with approximately equal numbers from industry and academia. The venue, the Hotel Ullensvang located on the Hardanger Fjord about $120 \mathrm{~km}$ from Bergen, turned out to be somewhat
The Hardanger Fjord from the Hotel Ullensvang where EIW- 6 was held.

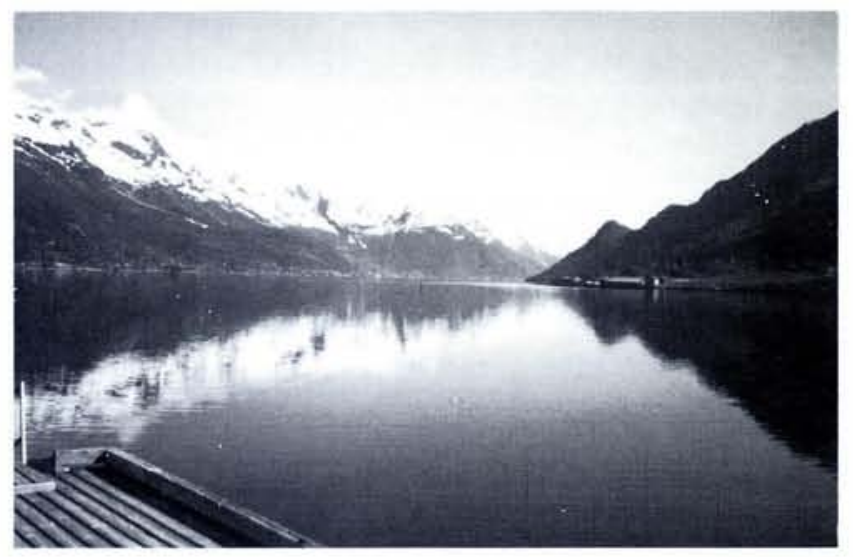

difficult to reach owing strikes at the local airlines, but this merely contributed to the excitement of the event.

The scientific program consisted of $21 / 2$ days of oral presentations and an evening poster session. Of the 17 speakers, 6 were from industry and 11 from academia. Tutorial lectures treating the basic physics and chemistry of conjugated polymers, both undoped as well as doped to a state of high electrical conductivity ("conducting" polymers), comprised about $1 / 3$ of the presenta- 
tions. Lectures aimed at more technological topics focused upon a wide and interesting array of present and potential applications, including: non-linear optical properties and integrated optics; semiconductor devices; sensors; integrated circuit processing; aerospace applications; textiles and highstrength fibre materials; organic batteries; molecular electronics; and various applications involving both low and high frequency electromagnetic responses. D.T. Clark closed the workshop with a positive look towards the future rôle of conjugated polymers within the chemical industry.

Particularly noteworthy among the industrial discussions were the three following items: researchers at IBM reported on the application of radiation-induced doped poIyaniline in high spatial resolution electroresists and electron beam discharge layers where conducting lines as small as $0.25 \mu \mathrm{m}$ have been produced. Lockheed, USA, has recently contracted for the large-scale production of polyaniline for use in conjunction with other polymers in the aerospace industry. Neste Corporation, Finland, has formed UNIAX, a California-based joint venture with a small consortium of American scientists prominent in the field of conjugated polymers. Representatives from several other industrial companies alluded to coming announcements about applications of "conducting polymers" but only a few could be pinned down.

W.R. Salaneck Linköping, Sweden

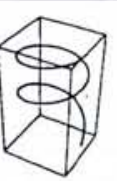

\section{Mathematica $^{\mathrm{TM}}$}

A System for Doing Mathematics by Computer

A Wolfram Research Inc. product

$\square$ Numerics - Works with numbers of arbitrary magnitude and precision.

Symbolics - Encyclopaedia of mathematical functions and operations used in arithmetic, algebra and analysis.

Procedural, functional and mathematical programming.

$\square$ Graphics - 2D, 3D and animated PostScript graphics.

Text processing - Fully interactive reports and textbooks.

$\square$ Runs on - MS-DOS based computers; Macintosh, Apollo, Hewlett Packard, IBM AIX/RT, MIPS, Silicon Graphics, Sony, Sun, VAX.

Now available in Europe from:

MathSoft Overseas, Inc.

POB 641, 1211 Geneva 3, Switzerland Tel. $++41(22) 465260$

Fax $++41(22) 465939$

\section{Hewlett-Packard Europhysics Prize}

The Hewlett-Packard Prize for 1991 has been awarded to Klaus Bechgaard of the H.C. Ørsted Institute, University of Copenhagen and to Denis Jérome, Laboratoire de Physique des Solides, Université de Paris-Sud, Orsay for the synthesis of a new class of organic metals and the discovery of their superconductivity and novel magnetic properties. The award ceremony will take place during the 11th Annual General Conference of the EPS Condensed Matter Division on 8-12 April 1991 in Exeter, UK.

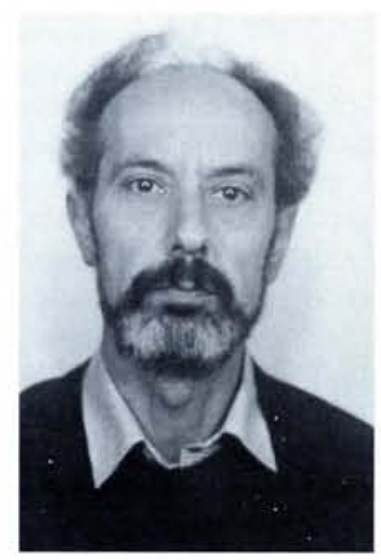

Denis Jérome, an accomplished musician and alpinist, is a Director of Research in the CNRS, France and leads the research group in organic conductors and superconductors at the Laboratoire de Physique des Solides, University of ParisSud, Orsay. He studied physics at the University of Paris, receiving his doctorate in 1965, before working as a postdoc at the University of California, La Jolla, USA. He moved back to Orsay in 1967 to head the high pressure group in Professor Friedel's laboratory. A Lauréat of the French Academy of Sciences in 1980 and winner of the 1985 Holweck Prize he became a correspondent of the Academy last year. Professor Jérome is the Chairman of the Publications Committee of the French Physical Society, serves as the Editor-in-Chief of the Journal de Physique and is a Member of the Executive Board of the Journal of Physics (Condensed Matter).

Klaus Bechgaard, an organic chemist, has been a Full Professor in the Department of General and Organic Chemistry, H.C. Orsted Institute, Copenhagen since 1989 following successive appointments, starting in 1969, as Research Assistant, Lecturer and Research Professor. After being awarded his doctorate from the University of Copenhagen in 1973, he became a postdoc at the John Hopkins University, USA. International collaboration continued with visiting positions in the USA (IBM, San Jose; University of Pennsylvania) and France (University of Paris-Sud; CNRS). Professor Bech-

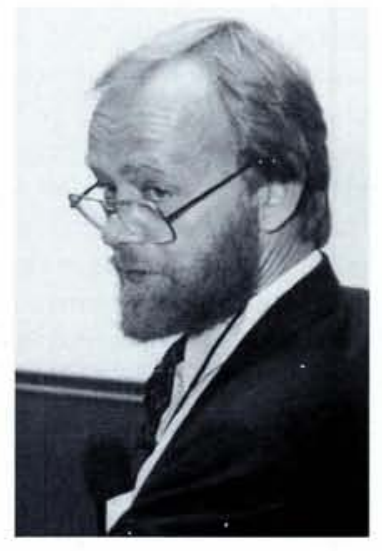
gaard won the B.S. Friedmann Award in 1986 and the I. Henriksen Award in 1987, has been a member of the Royal Danish Academy since 1984 and serves as a regional editor of Synthetic Metals.

Organic chemistry has had much success over the last half century in designing and fabricating materials with specific mechanical and optical properties, culminating in the 1970 's with the development of novel electronic and magnetic characteristics. There arose a unique cooperation between solid state physicists and organic chemists, one aim of whose work was to understand changes in physical properties in terms of molecular design: the prizewinners represent these two groups.

The organic compounds which have generated most interest comprise relatively small, planar molecules with weak intermolecular interactions that give rise to exotic new physical phenomena. Many of the compounds are also anisotropic, a feature that leads to effectively one-dimensional electronic characteristics in some classes of materials, and two-dimensional characteristics in others. The former have attracted considerable international interest, mainly because of the possibility of high-temperature superconductivity proposed by some workers. In 1964, W.A. Little at Stanford University argued that organic conductors with their one-dimensional conducting chains surrounded by easily polarizable molecules can generate the necessary couplings. $K$. Bechgaard and $\mathrm{D}$. Jérome published results six years later describing the first organic material to exhibit superconductivity.

Detailed analyses by many different workers have shown, however, that the superconducting state is not of the one-dimensional type proposed by Little, but is instead related to the classical BCS mechanism. Nonetheless, details of the superconducting state are influenced by the low-dimensional character of the compounds, and phenomena occurring above the superconducting transition temperature indicate the likely existence of fluctuations which can be interpreted as relating to one-dimensional superconductivity.

The one-dimensional character of the materials also produces a variety of other, by now well known, phenomena such as Peierls transitions, where the electronic charge and spin can be effectively decoupled to give separate transition temperatures for charge (metal-insulator) and spin (magnetic transition). The two-dimensional character found in some classes of organic conductors induces novel properties including, for example, the quantum Hall effect involving electrons moving in a plane.

K. Mortensen, J. Als-Nielsen Risø National Laboratory, Denmark 